\title{
NONLINEAR ANALYSIS OF THIN-WALLED FRAMES AND MEMBERS WITH ARBITRARY OPEN CROSS SECTIONS
}

\author{
By Tatsuro SAKIMOTO*, Toshitaka YAMAO**, \\ Ryosuke KIKUCHI*** and Tsutomu SAKATA****
}

\begin{abstract}
An incremental equilibrium equation for a beam-cloumn with arbitrary open cross sections is derived in a stiffness matrix form by using a moving element coordinate system and an incremental variational principle. A segmentation method for the cross section is used to analyze the development of plastic zones in the cross section and the effect of initial residual stresses arbitrarily distributed in the cross section. Yielding of the material is judged as a bi-axial stress problem under normal and shear stresses by using the flow theory of plasticity associated with von Mises yield criterion. Validity and efficiency of the present method are shown by illustrative examples.
\end{abstract}

\section{INTRODUCTION}

To analyze the behavior of metal frames or members upto the ultimate state is important for establishing design criteria and design procedures based on the ultimate state. A realistic solution to the problem for predicting the behavior of frames and members to the point of maximum load carrying capacity must include the effects of both large displacements and inelasticity of the material. Since formulations based on the finite element technique is effective to develop such a general method for analyzing combined nonlinear problems, efforts have been made to derive stiffness matrices for an inelastic beam-column element.

In an elastic beam theory, the shear center and neutral axes are chosen as the standard reference axes to simplify the formulation. But, after the start of yielding, these elastic reference axes lose their significance, because these axes continuously change in position and orientation along the member. Generally speaking, in the inelastic range, all the cross sectional properties must be determined as integrals of the area weighted not only by geometrical coordinates but also by material coefficients which describe relations between stresses and strains. Murray et al. ${ }^{1)}{ }^{2)}$ derived beam equations referred to two arbitrary points which are fixed to the cross section. By specializing these two points to the centroid and shear center, correspondence between derived equations and the classical elastic equations can be shown.

Extending this idea further, the writers have presented herein a set of beam equations referred to a single arbitrary point fixed to the cross section. If the correspondence to the classical beam equation is not necessary, this is more simple and easier formulation. In the present study, "incremental" equilibrium

* Member of JSCE, Dr. Eng., Professor, Department of Civil Engineering, Kumamoto University (Kurokame 2-chome, Kumamoto, 860)

** Member of JSCE, Ms. Eng., Research Associate, Department of Civil Engineering, Kumamoto University

*** Member of JSCE, Ms. Eng., Ministry of Construction, Formerly Graduate Student of Kumamoto University

**** Member of JSCE, Ms. Eng., Research Fellow, Department of Civil Engneering, Kumamoto University 
equations have been derived by using a moving element coordinate system and an incremental variational principle $^{3)}$. Murray et al. have derived the total equilibuium equations rather than the incremental ones because they have thought the basic equations may be considerably more complex than total equilibrium equations. Bui, so far as inelastic material responses are concerned, an incremental approach seems to be more effective, because the inelastic material behavior as described by the incremental theory of plasticity may have an incremental nature by itself.

Some of distinctive features provided in the present method are : a) Inelastic warping torsion of a member with arbitrary open cross section can be analyzed, b) Yielding of the material is judged as a bi-axial stress problem associated with normal and shear stresses, c) The stress distributions and development of the plastic zones in the cross section can be easily displayed at each incremental load step throughout the loading process, d) It is capable of accounting for the effect of strain reversal, and e) The effect of arbitrarily distributed residual stress can be considered.

\section{DERIVATION OF EQUILIBRIUM EQUATIONS}

\section{(1) Coordinate Systems and Assumptions}

The deformation of a body, of which strains are small despite its deflections are large, can be described by an incremental formulation with updated coordinates ${ }^{4) .5}$. Let us consider a member element at an arbitrary reference state $i$ on the way of a loading path. The local cartesian coordinates $(x, y, z)$ originated at an arbitrary reference point 0 in the cross section is set up and fixed to the element as shown in Fig. 1. The local coordinate system will be used as a Lagrangian frame for the subsequent state $i+1$. That is, the reference state $i$ is considered as an initially stressed state for determining the

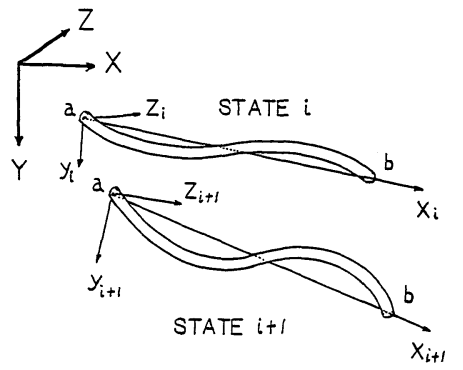

Fig. 1 Incremental moving coordinate systems. stresses, strains and displacements of the subsequent state $i+1$.

The basic assumptions used in the analysis are : a) A member has a thin-walled open cross section. b) The elastic-plastic strain-hardening behavior of the material can be modeled by the tri-linear stress-strain relationship between an equivalent stress and an equivalent strain. c) Plane sections remain plane under the influence of flexural moments (Bernoulli-Eulers' hypothesis) and warping functions used in the elastic theory of torsion is valid even after the initiation of yielding. d) The effect of shear stress and strain in the middle surface of the section wall due to bending or warping constraint may be small and negligible, e) The von Mises yield criterion is acceptable and the stress-strain relationship of Prandtl-Reuss is valid in the inelastic range, f) The distribution of shear strain caused by Saint Venant torsion is linear along the thickness of section plate even for a partially yielded cross section, g) The strain is small despite displacements are finite, h) Local buckling will not occur and distortion of the cross section shape may be negligible.

In the following derivation, the incremental quantities of displacements, forces, strains and stresses are denoted by the letters with bars and the total quantities of those are denoted by the letters without bars.

\section{( 2 ) Strain-Displacement Relationships}

Geometrical relations between the displacements and the rotation of the cross section are showm in Fig. 2. The axial strain increment of an arbitrary point $P(\eta, \zeta)$ on the cross section can be expressed by the displacement increments $(\bar{u}, \bar{v}, \bar{w})$ and the rotation increment $\bar{\phi}$ of the arbitrarily chosen reference point $\mathrm{O}$ as follows ${ }^{3)}$ :

$$
\bar{\varepsilon}=\bar{u}^{\prime}-(\eta-\zeta \bar{\phi}) \bar{v}^{\prime \prime}-(\zeta+\eta \bar{\phi}) \bar{w}^{\prime \prime}+\left[\left(\bar{v}^{\prime}\right)^{2}+\left(\bar{w}^{\prime}\right)^{2}\right] / 2+\left(\eta^{2}+\zeta^{2}\right)\left(\bar{\phi}^{\prime}\right)^{2} / 2+\omega \bar{\phi}^{\prime \prime}
$$

in which a prime denotes a derivative with respect to $x$ and $\omega$ denotes the normalized unit warping with respect to the point $O$. Since actual computations will be carried out by specializing the arbitrary point $O$ 
to the centroid of the cross section, the unit warping $\omega$ can be determined by an ordinary thin-walled beam theory. When the displacement increments are small, the axial strain increment is given by the ordinary linear equation,

$$
\bar{\varepsilon}=\bar{u}^{\prime}-\eta \bar{v}^{\prime \prime}-\zeta \bar{w}^{\prime \prime}+\omega \bar{\phi}^{\prime \prime}
$$

The shear strain increment $\bar{\gamma}$ due to St. Venant torsion is given by

$$
\bar{\gamma}=2 n \overline{\phi^{\prime}}
$$

in which $n$ is a coordinate originates at any point on the middle surface contour of the plate and is perpendicular to the coordinate $s$ which is a tangent to the middle surface contour (see Fig. 4).

\section{(3) Incremental Stress-Strain Relationship}

Only axial stress and shear stress due to St. Venant torsion will be considered for the yield condition in this analysis. The elastic-plastic strain-hardening behavior of the material in a bi-axial stress state is modeled by a tri-linear relation between an equivalent stress and an equivalent strain as shown in Fig. 3 . Adopting Hooke's law in the elastic range and assuming Prandtl-Reuss' equations and von Mises yield criterion in the inelastic range, we can derive the incremental stress-strain relationships as follows :

$$
\left[\begin{array}{c}
\bar{\sigma} \\
\bar{\tau}
\end{array}\right]=\left[\begin{array}{cc}
E\left(1-D_{1}\right) & -E D_{2} \\
-E D_{2} & G\left(1-D_{3}\right)
\end{array}\right]\left[\begin{array}{c}
\bar{\varepsilon} \\
\bar{\gamma}
\end{array}\right] \text { or } \sigma=\boldsymbol{D} \cdot \varepsilon
$$

in which $E$ and $G$ denote Young's and shear moduli of elasticity, respectively. The coefficients $D_{1}$ to $D_{3}$ are $D_{1}=D_{2}=D_{3}=0$ for the elastic range and for the inelastic range,

$$
\begin{aligned}
& D_{1}=E \sigma^{2} / 3 G B, \quad D_{2}=\sigma \tau / B, D_{3}=3 \tau^{2} / B \\
& B=(H / 3 G)\left(\sigma^{2}+3 \tau^{2}\right)+(E / 3 G) \sigma^{2}+3 \tau^{2}
\end{aligned}
$$

In the above, $H$ is a tangent slope of a stress versus plastic-strain diagram from a tensile coupon test (see Fig.3). For non-hardening material or for the plastic flow range $H$ equals to zero and for the strain-hardening range $H=E E_{s t} /\left(E-E_{s t}\right)$, in which $E_{s t}$ is the strain-hardening modulus.

The three stress-strain ranges, e. g. elastic, plastic and strain-hardening, can be distinguished by using the equivalent stress $\sigma_{E}$ and the equivalent plastic strain $\varepsilon_{E}^{p}$. That is, $\varepsilon_{E}^{p}=0$ and $\sigma_{E}<\sigma_{y}$ define the elastic range, $0<\varepsilon_{E}^{p}<\varepsilon_{s t}-\varepsilon_{y}$ and $\sigma_{E}=\sigma_{y}$ define the plastic range, and $\varepsilon_{E}^{p} \geqq \varepsilon_{s t}-\varepsilon_{y}$ and $\sigma_{E}=\sigma_{y s t}$ define the strain-hardening range, in which $\sigma_{y s t}=\sigma_{y}+H\left(\varepsilon_{E}^{p}-\varepsilon_{s t}+\varepsilon_{y}\right)$ (see Fig. 3).

The equivalent stress and the equivalent plastic strain are defined herein as follows ${ }^{5)}$ :

$$
\sigma_{E}=\sqrt{\sigma^{2}+3 \tau^{2}}, \quad \varepsilon_{E}^{p}=\sum \bar{\varepsilon}_{E}^{p}=\sum 2 \sigma_{E} \lambda / 3
$$

in which the summation must be taken for each incremental load step. The positive coefficient which has been introduced in Prandtl-Reuss' equations can be calculated by

$$
\lambda=[(E \sigma / 2 G) \bar{\varepsilon}+(3 \tau / 2) \bar{\gamma}] /\left(H \sigma_{E}^{2} / 3 G+E \sigma^{2} / 3 G+3 \tau^{2}\right)
$$

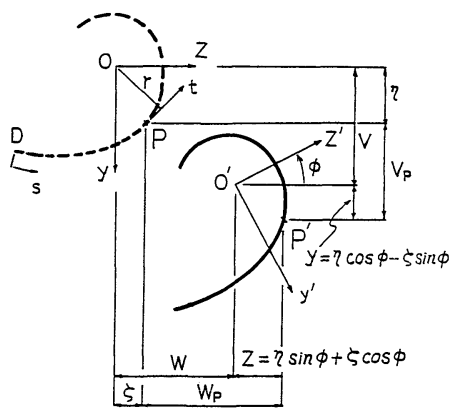

Fig. 2 Displacements of cross section.

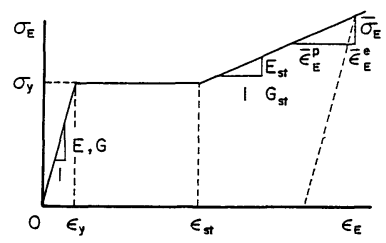

(A) EOUIVALENT STRESS VS. TOTAL STRAIN DIAGRAM

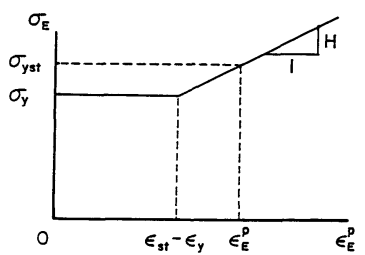

(B) EQUIVALENT STRESS VS. PLASTIC STRAIN DIAGRAM

Fig. 3 Tri-linear stress-strain relation. 
In the numerical calculation, $\lambda, \sigma_{E}, \varepsilon_{E}^{p}$ are evaluated from Eqs. 5 and 6 by using the stresses, strains and strain increments of the present state $i$ and elastic, plastic and strain-hardening states are judged for each cross-sectional segment and then next incremental stresses are evaluated by Eq. 4.

(4) Tangent Stiffness of a Partially Yielded Cross Section

The relationships between the increments of the resultant forces $r^{T}=\left(\bar{N},-\bar{M}_{z}, \bar{M}_{y}, \bar{M}_{\omega}, \bar{T}\right)$ and the corresponding deformation increments $\boldsymbol{d}^{T}=\left(\bar{u}^{\prime},-\bar{v}^{\prime \prime},-\bar{w}^{\prime \prime}, \bar{\phi}^{\prime \prime}, \bar{\phi}^{\prime}\right)$ are to be derived for a partially yielded cross section of unit length shown in Fig. 4. The increments of the resultant forces may be calculated by the integration of stress increments over the cross sectional area, A. Those are,

$$
\begin{aligned}
& \bar{N}=\int \bar{\sigma} d A, \bar{M}_{z}=-\int \eta \bar{\sigma} d A, \bar{M}_{y}=\int \zeta \bar{\sigma} d A, \\
& \bar{M}_{\omega}=\int \omega \bar{\sigma} d A, \bar{T}=2 \int n \bar{\tau} d A
\end{aligned}
$$

In a matrix notation,

$$
r=\int C^{T} \sigma d A
$$

in which the superscript $T$ stands for a transposition of the matrix and

$$
C=\left[\begin{array}{ccccc}
1 & \eta & \zeta & \omega & 0 \\
0 & 0 & 0 & 0 & 2 n
\end{array}\right]
$$

From Eqs. (2) and (3), the relation between the strain increments and the deformation increments can be written as

$$
\varepsilon=C \cdot d
$$

Substitution of Eqs. (4) and (9) into Eq. (8) yields

$$
\boldsymbol{r}=\int \boldsymbol{C}^{T} \boldsymbol{D C d} d A=\left[\int \boldsymbol{C}^{T} \boldsymbol{D C} d A\right] \cdot \boldsymbol{d}=\boldsymbol{S} \cdot \boldsymbol{d}
$$

This matrix $S$ represents the tangent stiffness of a partially yielded member of unit length. Upper right half elements $S_{i j}$ of this symmetric $5 \times 5$ matrix are given as follows:

$$
\begin{aligned}
& S_{11}=E \int\left(1-D_{1}\right) d A, \quad S_{12}=E \int \eta\left(1-D_{1}\right) d A, \quad S_{13}=E \int \zeta\left(1-D_{1}\right) d A, \\
& S_{14}=E \int \omega\left(1-D_{1}\right) d A, \quad S_{15}=-2 E \int n D_{2} d A, \quad S_{22}=E \int \eta^{2}\left(1-D_{1}\right) d A, \\
& S_{23}=E \int \eta \zeta\left(1-D_{1}\right) d A, \quad S_{24}=E \int \eta \omega\left(1-D_{1}\right) d A, \quad S_{25}=-2 E \int n \eta D_{2} d A, \\
& S_{33}=E \int \zeta^{2}\left(1-D_{1}\right) d A, \quad S_{34}=E \int \zeta \omega\left(1-D_{1}\right) d A, \quad S_{35}=-2 E \int \eta \zeta D_{2} d A, \\
& S_{44}=E \int \omega^{2}\left(1-D_{1}\right) d A, \quad S_{45}=-2 E \int n \omega D_{2} d A, \quad S_{55}=4 G \int n^{2}\left(1-D_{3}\right) d A .
\end{aligned}
$$

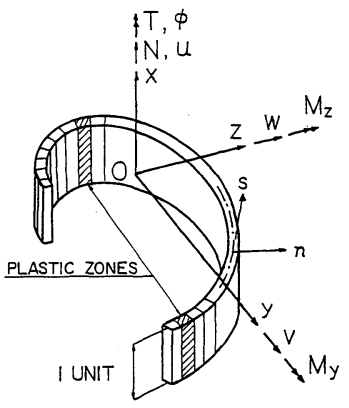

Fig. 4 Positive directions of resultant forces and displacements.

\section{(5) Governing Equations of Equilibrium}

When a member, starting from the initial state $i$ prestressed by the total external load $p$, reaches to the subsequent state $i+1$ after producing the displacement increment $\overline{\boldsymbol{u}}_{X}$ caused by the load increment $\overline{\boldsymbol{p}}$, the incremental potential energy $V$ during the deformation is written as follows:

$$
V=-\left[\left(\overline{\boldsymbol{u}}_{X}+\boldsymbol{u}_{X}\right)^{T}(\overline{\boldsymbol{p}}+\boldsymbol{p})-\boldsymbol{u}_{X}^{T} \boldsymbol{p}\right]=-\overline{\boldsymbol{u}}_{X}^{T}(\overline{\boldsymbol{p}}+\boldsymbol{p})-\boldsymbol{u}_{X}^{T} \overline{\boldsymbol{p}}
$$

Assuming the linearity during an incremental step, the incremental strain energy $U$ to be stored in the member element of length $l$ is :

$$
U=\frac{1}{2} \int_{0}^{l} \boldsymbol{d}^{T} \boldsymbol{r} d x+\int_{0}^{l}\left(\int_{A} \sigma \bar{\varepsilon} d A\right) d x+\int_{0}^{l}\left(\int_{A} \tau \bar{\gamma} d A\right) d x
$$

Substitution of Eqs. (1), (3) and (10) into this equation yields

$$
\begin{aligned}
& U=\frac{1}{2} \int_{0}^{l} \boldsymbol{d}^{T} \boldsymbol{S} \boldsymbol{d} d x+\int_{0}^{l} N\left[\bar{u}^{\prime}+\frac{1}{2}\left(\bar{v}^{\prime}\right)^{2}+\frac{1}{2}\left(\bar{w}^{\prime}\right)^{2}\right] d x+\int_{0}^{l} M_{z}\left(\bar{v}^{\prime \prime}+\bar{\phi} \bar{w}^{\prime \prime}\right) d x \\
& -\int_{0}^{l} M_{y}\left(\bar{w}^{\prime \prime}-\bar{\phi} \bar{v}^{\prime \prime}\right) d x+\int_{0}^{l} K(\bar{\phi})^{2} d x+\int_{0}^{l} M_{\omega} \bar{\phi}^{\prime \prime} d x+\int_{0}^{l} T \bar{\phi}^{\prime} d x
\end{aligned}
$$


in which $N, M_{z}, M_{y}, M_{\omega}$ and $T$ are total resultant forces defined by similar equations to Eq. ( 7 ) and $K=$ $\int \sigma\left(\eta^{2}+\zeta^{2}\right) d A$

Since satisfactory information about the displacement functions for an elasto-plastic member element is not available so far, polynomials, which are usually used in an elastic finite element procedure, are also employed herein. Namely $\bar{u}$ and $\bar{v}, \bar{w}, \bar{\phi}$ are represented by linear and cubic polynomials of $x$, respectively. Resultant forces $N$ and $K$ are considered to be constant along $x$-axis and $M_{z}, M_{y}, M_{\omega}$ and $T$ are assumed to be linear polynomials of $x$. Then, using the ordinary techniques applied in the finite element method, Eq. (14) can be expressed in terms of the increments of nodal displacements and resultant forces determined at the associated nodes. After all, the increment of the potential energy $\pi=U+V$ can be written in terms of the increments of the nodal displacement $u$.

Since the stationary condition of $\pi$ gives an incremental equilibrium condition ${ }^{6)}$, first partial derivatives of $\pi$ with respect to $\bar{u}$ yield the following incremental equilibrium equation :

$\left(\boldsymbol{k}_{e p}+\boldsymbol{k}_{g}\right) \cdot \overline{\boldsymbol{u}}=\overline{\boldsymbol{p}}+(\boldsymbol{p}-\boldsymbol{f})$

in which the displacement vector and the force vectors are given as follows:

$$
\begin{aligned}
& \overline{\boldsymbol{u}}=\left[\begin{array}{llllllllllllll}
\bar{u}_{a} & \bar{v}_{a} & \bar{w}_{a} & \bar{\phi}_{a} & \bar{w}_{a}^{\prime} & \bar{v}_{a}^{\prime} & \bar{\phi}_{a}^{\prime} & \bar{u}_{b} & \bar{v}_{b} & \bar{w}_{b} & \bar{\phi}_{b} & \bar{w}_{b}^{\prime} & \bar{v}_{b}^{\prime} & \bar{\phi}_{b}^{\prime}
\end{array}\right]^{T} \\
& \overline{\boldsymbol{p}}=\left[\begin{array}{llllllllllllll}
\bar{P}_{x a} & \bar{P}_{y a} & \bar{P}_{z a} & \bar{M}_{x a} & \bar{M}_{y a} & \bar{M}_{z a} & \bar{M}_{\omega a} & \bar{P}_{x b} & \bar{P}_{y b} & \bar{P}_{z b} & \bar{M}_{x b} & \bar{M}_{y b} & \bar{M}_{z b} & \bar{M}_{\omega b}
\end{array}\right]^{T} \\
& \boldsymbol{p}=\left[\begin{array}{llllllllllllll}
P_{x a} & P_{y a} & P_{z a} & M_{x a} & M_{y a} & M_{z a} & M_{\omega a} & P_{x b} & P_{y b} & P_{z b} & M_{x b} & M_{y b} & M_{z b} & M_{\omega b}
\end{array}\right]^{T} \\
& \boldsymbol{f}=\left[\begin{array}{lll}
N_{a}+N_{b} \\
2
\end{array}-\frac{M_{z a}-M_{z b}}{l} \frac{M_{y a}-M_{y b}}{l}-\frac{M_{\omega a}-M_{\omega b}}{l}-T-M_{y a}-M_{z a}-M_{\omega a}-\frac{\left(T_{b}-T_{a}\right) l}{12}\right. \\
& \left.\frac{N_{a}+N_{b}}{2} \frac{M_{z a}-M_{z b}}{l} \frac{-M_{y a}+M_{y b}}{l} \frac{M_{\omega a}-M_{\omega b}}{l}+T \quad M_{y b} \quad M_{z b} \quad M_{\omega b}+\frac{\left(T_{b}-T_{a}\right) l}{12}\right]^{T}
\end{aligned}
$$

In these equations, subscripts $a$ and $b$ denote the quantities related to the nodes $a$ and $b$, respectively. The details of the stiffness matrices $\boldsymbol{k}_{e p}$ and $\boldsymbol{k}_{g}$ are shown in Appendix. The matrix $\boldsymbol{k}_{e p}$ represents the stiffness of an elasto-plastic member. The matrix $\boldsymbol{k}_{g}$ is an initial stress matrix which represents geometrical nonlinearity caused by finite displacements. The term $(\boldsymbol{p}-\boldsymbol{f})$ in Eq. (15) is considered as unbalanced forces caused either by the linearization assumed in the formulation and by yielding of the material occurred on the half way of an incremental load step. In order to eliminate these unbalanced forces, an iterative procedure is needed for numerical solutions. Transformation of Eq. (15) to the global coordinate system by the ordinary transformation matrix $T$ yields

$$
\left[\boldsymbol{K}_{e p}+\boldsymbol{K}_{g}\right] \cdot \bar{U}=\bar{P}+(\boldsymbol{P}-\boldsymbol{T} \cdot \boldsymbol{f})
$$

Equations obtained by assembling Eq. (17) for a whole structural system are the governing equilibrium equations to be solved.

\section{(6) Computation Procedure}

In order to analyze the development of plastic zones, the structure is divided into a number of member elements and cross sectional segments as shown in Fig. 5. Strains and stresses are evaluated at the central points of the cross sectional segments and added to the residual strains and stresses which have been given prior to the loading. Cross sectional quantities and resultant forces used in the stiffness matrices are determined by a numerical integration at the end sections of the member elements. This segmentation for the cross section allows to analyze arbitrary shapes of cross sections with arbitrary residual strain distributions. The Newton-Raphson procedure is employed for the iterative computation in solving Eq. $(17)^{3)}$. Descriptions are omitted herein, but the computer program allows transverse loads applied at any points in the plane of a cross section.

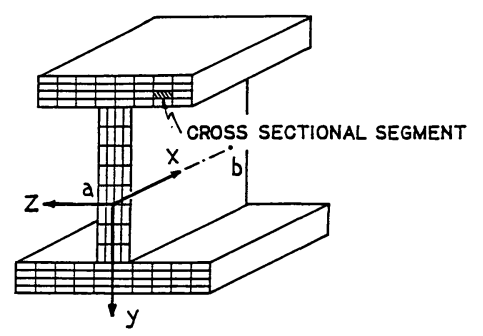

Fig. 5 Discretization of structural system. 


\section{NUMERICAL EXAMPLES}

Two example for which experimental or theoretical results are available were selected to examine the applicability of the present method.

The first example is the Elastica for which the analytical solution by the elliptic integration is available ${ }^{7}$. The theoretical model is shown in the inset of Fig. 6. In the present analysis, the column is divided into 10 longitudinal elements and 192 cross sectional segments ( 8 divisions in width or height and 8 layers in the thickness for both flange and web plates). Fig. 6 shows the load versus displacements diagrams in the non-dimensional form. The results of the present method correspond to the analytical solution fairly well. As is known, most of large displacement analyses require a double precision computation (64 bits for one word in a digital computer) and some numerical techniques to remove the displacements caused by the rigid body motion from the total displacements in calculating strains from the computed displacements. It will be worth to emphasize that the present computation is carried out by single precision (32 bits for one word in the digital computer) without using any such numerical technique to remove the displacements caused by the rigid body motion. Therefore, the method can be easily applied to inelastic analysis for complex structures.

As the next example, a comparison is made with the finite element solution by Murray et al. ${ }^{8}$, and the experimental results of Birnstiel ${ }^{9)}$ for an inelastic beamcolumn subjected to biaxial bending and compression. The theoretical model solved by this method is shown in the inset of Fig. 7. Its structural and material properties follow those of Ref. 9, as closely as possible. However, the residual stresses distribution and the value of the yield stress $\sigma_{y}=$ $34.5 \mathrm{ksi}$ (238 Mpa) are assumed to follow those of Ref. 8. In the present method the beam-column is divided into the same as the first example (10 longitudinal elements and 192 cross sectional segments). The eccentric axial load is converted to a centroidal load and bi-axial end moments. Rotation and transverse displacements at midheight are shown in Fig. 7. The decending part of the curve after the peak load is not shown in the figure, because the present computation is stopped when trivial large displacements caused by zero stiffness would take place.

Agreement in these three results is fairly well and efficiency of the present method is confirmed.

\section{CONCLUSION}

A theoretical formulation and a finite element procedure for three-dimensional large displacement behavior of inelastic frames and

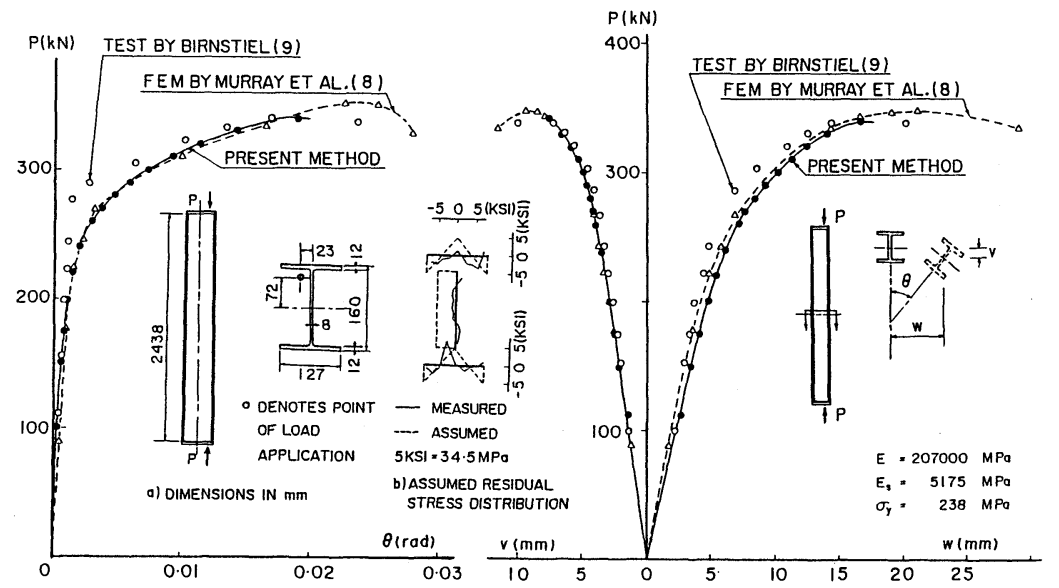

Fig. 7 Rotation and transverse desplacements at midheight for biaxial bending of H-column. 
members with arbitrary thin-walled open cross section have been presented. The illustrative examples investigated in order to test the validity and applicability of the present method show that results obtained have the desired accuracy and efficiency comparing with available theorical or experimental results. By this method it is now capable to carry out parametric studies to establish design criteria and design procedures for complex metal structures which will be controlled by inelastic instability under realistic loadings and material response.

The computations for examples were conducted by the digital computer FACOM M-382 of the Computer Center of Kyushu University.

Appendix Elements of Stiffness Matrices $\boldsymbol{k}_{e p}$ and $\boldsymbol{k}_{g}$ (showing upper right half only)

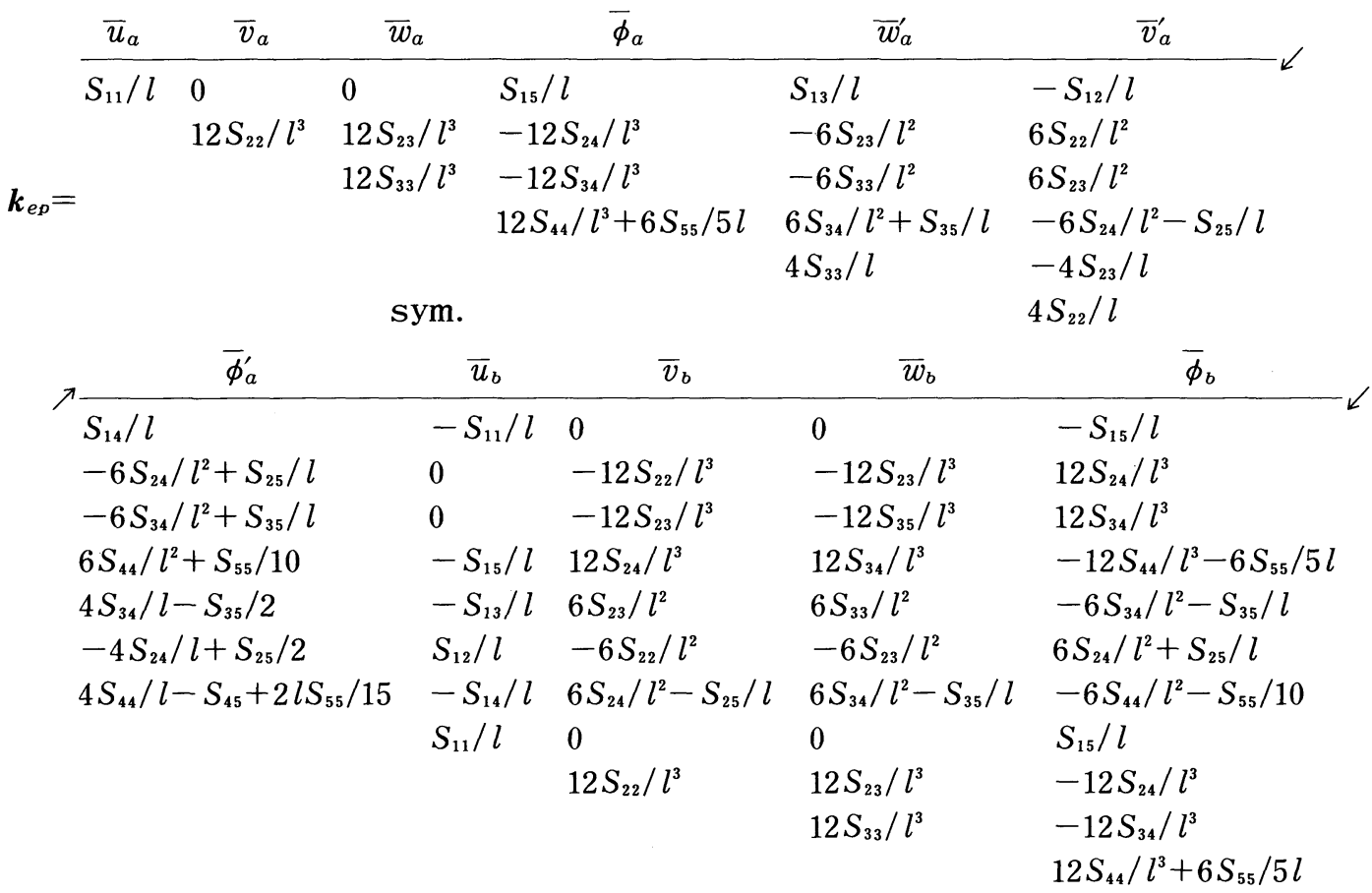

sym.

\begin{tabular}{lll}
\multicolumn{1}{c}{$\bar{w}_{b}^{\prime}$} & \multicolumn{1}{c}{$\bar{v}_{b}^{\prime}$} & \multicolumn{1}{c}{$\bar{\phi}_{b}^{\prime}$} \\
\hline$-S_{13} / l$ & $S_{12} / l$ & $-S_{14} / l$ \\
$-6 S_{22} / l^{2}$ & $6 S_{22} / l^{2}$ & $-6 S_{24} / l^{2}-S_{25} / l$ \\
$-6 S_{33} / l^{2}$ & $6 S_{23} / l^{2}$ & $-6 S_{34} / l^{2}-S_{35} / l$ \\
$6 S_{34} / l^{2}-S_{35} / l$ & $-6 S_{24} / l^{2}+S_{25} / l$ & $6 S_{44} / l^{2}+S_{55} / 10$ \\
$2 S_{33} / l$ & $-2 S_{23} / l$ & $2 S_{34} / l+S_{35} / 2$ \\
$-2 S_{23} / l$ & $2 S_{22} / l$ & $-2 S_{24} / l-S_{25} / 2$ \\
$2 S_{34} / l-S_{35} / 2$ & $-2 S_{24} / l+S_{25} / 2$ & $2 S_{44} / l-l S_{55} / 30$ \\
$S_{13} / l$ & $-S_{12} / l$ & $S_{14} / l$ \\
$6 S_{23} / l^{2}$ & $-6 S_{22} / l^{2}$ & $6 S_{24} / l^{2}+S_{25} / l$ \\
$6 S_{33} / l^{2}$ & $-6 S_{23} / l^{2}$ & $6 S_{34} / l^{2}+S_{35} / l$ \\
$-6 S_{34} / l^{2}+S_{35} / l$ & $6 S_{24} / l^{2}-S_{25} / l$ & $-6 S_{44} / l^{2}-S_{55} / 10$ \\
$4 S_{33} / l$ & $-4 S_{23} / l$ & $4 S_{34} / l+S_{35} / 2$ \\
& $4 S_{22} / l$ & $-4 S_{24} / l-S_{25} / 2$ \\
& & $4 S_{44} / l+S_{45}+2 l S_{55} / 15$
\end{tabular}




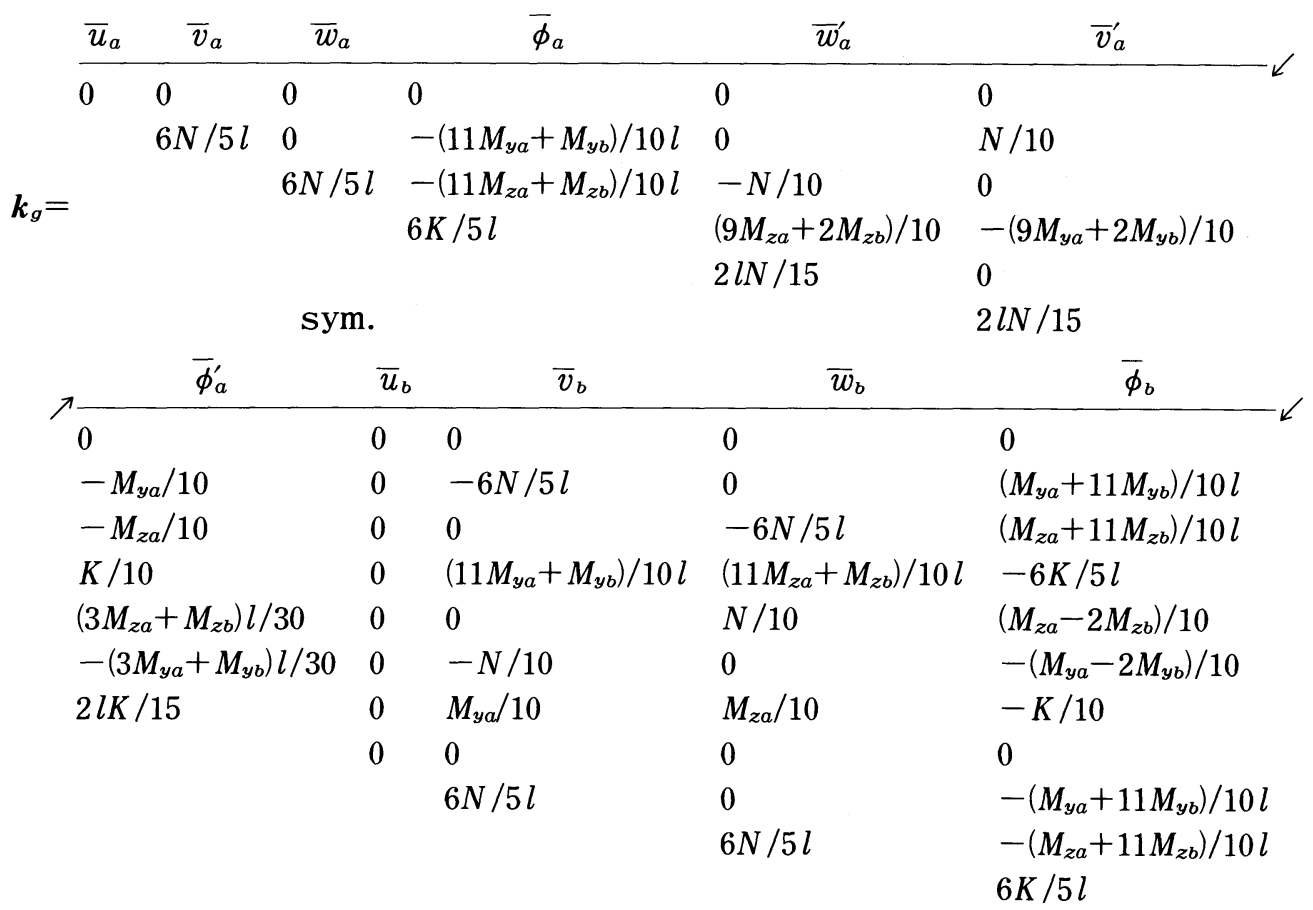

sym.

\begin{tabular}{lll}
$\nearrow$ & \multicolumn{1}{c}{$\bar{w}_{b}^{\prime}$} & \multicolumn{1}{c}{$\bar{\phi}_{b}^{\prime}$} \\
\hline 0 & 0 & 0 \\
0 & $N / 10$ & $-M_{y b} / 10$ \\
$-N / 10$ & 0 & $-M_{z b} / 10$ \\
$\left(2 M_{z a}-M_{z b}\right) / 10$ & $-\left(2 M_{y a}-M_{y b}\right) / 10$ & $K / 10$ \\
$-l N / 30$ & 0 & $-l M_{z a} / 30$ \\
0 & $-l N / 30$ & $l M_{y a} / 30$ \\
$-l M_{z b} / 30$ & $l M_{y b} / 30$ & $-l K / 30$ \\
0 & 0 & 0 \\
0 & $-N / 10$ & $M_{y b} / 10$ \\
$N / 10$ & 0 & $M_{z b} / 10$ \\
$-\left(2 M_{z a}+9 M_{z b}\right) / 10$ & $\left(2 M_{y a}+9 M_{y b}\right) / 10$ & $-K / 10$ \\
$2 l N / 15$ & 0 & $\left(M_{z a}+3 M_{z b}\right) l / 30$ \\
& $2 l N / 15$ & $-\left(M_{y a}+3 M_{y b}\right) l / 30$ \\
& & $2 l K / 15$
\end{tabular}

in which

$N=\left(N_{a}+N_{b}\right) / 2$

\section{REFERENCES}

1) Murray, D. W. and Rajasekanan, S. : Technique for Formulating Beam Equation, Proc. of ASCE, Vol. 101, No. EM 5, pp. 561 —573, Oct., 1975.

2) Rajasekanan, S. and Murray, D. W. : Finite Element Solution of Inelastic Beam Equations, Proc. of ASCE, Vol. 99, No. ST 6 , pp. 1025 1041, June, 1973.

3) Komatsu, S. and Sakimoto, T. : Nonlinear Analysis of Spatial Frames Consisting of Members with Closed Cross-Sections, Proc. of JSCE, No. 252, pp. 143 157, Aug., 1976.

4) Hofmeister, L. D., Greenbaum, G. A. and Evensen, D. A. : Large Strain Elasto-Plastic Finite Element Analysis, Proc. of 
AIAA/ASME, pp. 1248 1254, July, 1971.

5) Yamada, Y. : Sosei Rikigaku, Nikkan Kougyo Shinbunsha, 1960.

6) Reckling, G. -A. : Plastizitäts Theorie und ihre Anwendung auf Festigkeitzprobleme, Springer-Verlag, Berlin, pp. 27 62, (in German) 1967.

7) Timoshenko, S. P. and Gere, J. M. : Theory of Elastic stability, 2nd ed., Mcgraw-Hill Book. Co., Inc., pp. 76 82, 1961.

8) Epstein, M., Nixon, D. and Murray, D. W. : Large Displacement Inelastic Analysis of Beam-Columns, Proc. of ASCE, Vol. 104, No. ST 5, pp. 841 853, May, 1978.

9) Birnstiel, C. : Experiments on H-columns under Biaxial Bending, Proc. of ASCE, Vol.94, No. ST 10, pp. 2429 2449, April, 1968.

(Roceived April 13 1985) 